\title{
Focal hemodynamic patterns of status epilepticus detected by susceptibility weighted imaging (SWI)
}

\author{
Jerome Aellen • Eugenio Abela $\cdot$ Sarah E. Buerki • Raimund Kottke $\cdot$ Elisabeth Springer • \\ Kaspar Schindler • Christian Weisstanner • Marwan El-Koussy • Gerhard Schroth • \\ Roland Wiest • Jan Gralla • Rajeev K. Verma
}

Received: 5 December 2013 /Revised: 22 May 2014 / Accepted: 24 June 2014 / Published online: 6 August 2014

(C) European Society of Radiology 2014

\begin{abstract}
Objective To investigate pathological findings in the susceptibility weighted imaging (SWI) of patients experiencing convulsive (CSE) or non-convulsive status epilepticus (NCSE) with focal hyperperfusion in the acute setting.

Methods Twelve patients (six with NCSE confirmed by electroencephalogram (EEG) and six patients with CSE with seizure event clinically diagnosed) underwent MRI in this acute setting (mean time between onset of symptoms and MRI was $3 \mathrm{~h} 8 \mathrm{~min}$ ), including SWI, dynamic susceptibility contrast MR imaging (DSC) and diffusion-weighted imaging (DWI). MRI sequences were retrospectively evaluated and compared with EEG findings (10/12 patients), and clinical symptoms.

Results Twelve out of $12(100 \%)$ patients showed a focal parenchymal area with pseudo-narrowed cortical veins on SWI, associated with focal hyperperfused areas (increased cerebral blood flow (CBF) and mean transit time (MTT) shortening), and cortical DWI restriction in 6/12 patients $(50 \%)$. Additionally, these areas were associated with ictal or postical EEG patterns in 8/10 patients (80\%). Most frequent acute clinical findings were aphasia and/or hemiparesis in eight patients, and all of them showed pseudo-narrowed
\end{abstract}

J. Aellen $\cdot$ R. Kottke $\cdot$ E. Springer $\cdot$ C. Weisstanner $\cdot$ M. El-Koussy $\cdot$

G. Schroth $\cdot$ R. Wiest $\cdot$ J. Gralla $\cdot$ R. K. Verma $(\bowtie)$

University Institute for Diagnostic and Interventional

Neuroradiology, University Hospital Bern and Inselspital, University

of Bern, Freiburgstrasse 4, 3010 Bern, Switzerland

e-mail: rajeev.verma@insel.ch

E. Abela $\cdot$ K. Schindler

Department of Neurology, Inselspital, University of Bern, Bern,

Switzerland

S. E. Buerki

Department of Neuropaediatrics, University Children's Hospital, Inselspital, Bern, Switzerland veins in those parenchymal areas responsible for these symptoms.

Conclusion In this study series with CSE and NCSE patients, SWI showed focally pseudo-narrowed cortical veins in hyperperfused and ictal parenchymal areas. Therefore, SWI might have the potential to identify an ictal region in CSE/ NCSE.

Key Points

- The focal ictal brain regions show hyperperfusion in DSC MR-perfusion imaging.

- SWI shows focally diminished cortical veins in hyperperfused ictal regions.

- SWI has the potential to identify a focal ictal region in CSE/ NCSE.

Keywords Magnetic resonanceimaging P Perfusion imaging · Susceptibility-weighted imaging (SWI) - Status epilepticus · Non-convulsive status epilepticus

\section{Introduction}

The definition of a status epilepticus (SE) varies slightly among authors but is commonly accepted as a single unremitting seizure extraordinarily prolonged lasting longer than 5 min ('impending SE') or 30 min ('established SE') or repetitive clinical seizures without an interictal return to the baseline clinical state $[1,2]$. SE may lead to a progressing and deepening impairment of consciousness and may become life threatening (mortality rates up to $18 \%$ have been described [3]). While several typical clinical findings can occur in convulsive SE (CSE), i.e. prolonged focal or generalized myoclonus, a non-convulsive status epilepticus (NCSE) may present with much subtler and less specific signs. Indeed, NCSE is a heterogeneous disorder with multiple subtypes, e.g. absence status epilepticus, simple partial and complex partial status 
epilepticus or subtle status epilepticus [4]. Electroencephalogram (EEG), the diagnostic gold standard, may not be available in emergency situations in due time. Therefore the clinical diagnosis remains challenging, and NCSE is known to be underdiagnosed [5]. Recently, magnetic resonance imaging (MRI) and computed tomography (CT) have emerged as promising tools to help in diagnosing CSE and NCSE in the acute setting. In a few studies, a focal hyperperfusion of the affected cerebral region was demonstrated [6-11]. Additionally, the affected regions partly showed a diffusion restriction in some studies, presumably because the compensatory mechanism of hyperperfusion is insufficient to prevent the stimulation of anaerobic glycolysis due to ictal activity leading to apparent diffusion coefficient (ADC) reductions $[10,12,13]$. However, susceptibility-weighted imaging (SWI) has not yet been evaluated in detail for SE. As a useful diagnostic sequence, SWI has been widely applied, e.g. for detecting or evaluating intracranial haemorrhage, calcification, cerebral venous thrombosis, tumour evolution $[14,15]$ or haemorrhagic transformation of stroke [16-18], using the parametric properties of deoxyhaemoglobin. SWI has been described inter alia to be helpful in detecting occult calcified lesions or vascular malformations that may be responsible for seizures [19]. The role of SWI has not yet been evaluated in the acute ictal setting with respect to alterations in cortical vein appearance due to perfusion changes. It is assumed that the deoxyhaemoglobin content in veins correlates with their prominence and darkness in SWI due to their paramagnetic properties. Focusing on cortical veins in SWI, their prominent and dark appearance has been described in stroke patients in perfusion-disturbed regions after thromboembolic occlusion [17, 18, 20, 21], owing to increased deoxyhaemoglobin levels, secondary to an increased extraction fraction of oxygen by the ischemic parenchyma. Further, prominent-appearing cortical veins have been described in patients with hemiplegic migraine correlating with hypoperfusion [22, 23]. Since focal parenchymal areas are expected to be hyperperfused during seizure activity, an opposite effect with correlating pseudo-narrowing of cortical veins would be expected in case of a lower deoxyhaemoglobin content, and therefore decreased paramagnetic properties. To our knowledge only one case report by Lee et al. [24] has described this phenomenon. Our retrospective study was undertaken to investigate whether pseudo-narrowed or pseudo-diminished cortical veins in SWI are a finding that could be diagnostically useful for detecting ictal activity. Further, we evaluated a presumed correlation between the location of pseudo-narrowed cortical veins in SWI with findings in DWI, DSC, EEG and clinical symptoms.

\section{Materials and methods}

\section{Patient data}

The study was approved by the local ethics committee. Inclusion criteria for this retrospective study were a clinically or electrophysiologically confirmed CSE or NCSE (NCSE selected cases were all by definition EEG-proven). Mandatory MRI sequences were DSC, DWI and SWI sequences according to the domestic emergency/stroke protocol and showing a focal decreased mean transit time (MTT) in DSC in the colour-coded maps. Patient data were excluded if image quality was poor, e.g. as a result of motion artefacts. A total of 12 patients (eight female and four male; age range, 1-87 years; mean, 34.5 years) fulfilled these criteria. Ten of 12 subjects had received EEG in between $47 \mathrm{~min}$ and $31 \mathrm{~h}$ 38 min after symptom onset, and prior or after MRI (time range $4 \mathrm{~h} 15 \mathrm{~min}$ before and $21 \mathrm{~h} 8 \mathrm{~min}$ after MRI acquisition).

Patients were referred to our neuroradiology department from the emergency department where they had been evaluated by neurologists. They were referred for acute neurological symptoms and/or to rule out stroke. Five of 12 patients were intubated (four children and one adult).

\section{Data acquisition}

Imaging studies were performed using a $1.5-\mathrm{T}$ and a 3-T Siemens system (Magnetom Avanto, Magnetom Trio, respectively; Siemens Medical Solution, Erlangen, Germany) with a 12-channel head coil. The MRI protocol performed was our local emergency/stroke protocol, which includes the following sequences: axial DWI, axial T2 SE, ToF angiography, perfusion imaging, contrast-enhanced angiography of the cervical and intracranial arteries, and an axial T1 SE postcontrast. For the 1.5-T MRI the SWI parameters were TR $49 \mathrm{~ms}$, TE $40 \mathrm{~ms}$, number of averages 1, FoV read $230 \mathrm{~mm}$, FoV phase $81.3 \%$, voxel size $1.1 \times 0.9 \times 1.8 \mathrm{~mm}$, flip angle $15^{\circ}$, acquisition time $2: 59 \mathrm{~min}$. Perfusion imaging parameters (DSC) were TR $1,410 \mathrm{~ms}$, TE $30 \mathrm{~ms}$, number of averages 1 , FoV read $230 \mathrm{~mm}$, FoV phase $100 \%$, voxel size $1.8 \times 1.8 \times$ $5.0 \mathrm{~mm}$, flip angle $90^{\circ}$, acquisition time 2:00 min. DWI parameters were TR $3,000 \mathrm{~ms}$, TE $89 \mathrm{~ms}$, number of averages 4, FoV read $230 \mathrm{~ms}$, FoV phase $100 \%$, voxel size $1.2 \times 1.2 \times$ $5.0 \mathrm{~mm}$, acquisition time 1:35 min. For the 3-T scanner the SWI parameters were as follows: TR $27 \mathrm{~ms}$, TE $20 \mathrm{~ms}$, number of averages $1, \mathrm{FoV}$ read $230 \mathrm{~mm}$, FoV phase $75.0 \%$, voxel size $0.9 \times 0.9 \times 2.0 \mathrm{~mm}$, flip angle $15^{\circ}$, acquisition time $2: 59 \mathrm{~min}$. A standard perfusion imaging sequence was used with following parameters (DSC): TR 1,400 ms, TE $29 \mathrm{~ms}$, number of averages 1, FoV read $230 \mathrm{~mm}$, FoV phase 
Table 1 MRI parameter overview

\begin{tabular}{lccllll}
\hline & TR $(\mathrm{ms})$ & TE $(\mathrm{ms})$ & FOV $(\mathrm{mm})$ & Voxel size $(\mathrm{mm})$ & Flip angle $\left(^{\circ}\right)$ & $\begin{array}{c}\text { Acquisition } \\
\text { time }(\mathrm{min})\end{array}$ \\
\hline Sequence $@ 1.5 \mathrm{~T}$ & & & & & \\
SWI & 49 & 40 & 230 & $1.1 \times 0.9 \times 1.8$ & 15 & $02: 59$ \\
DSCE & 1,410 & 30 & 230 & $1.2 \times 1.2 \times 5$ & 90 & $02: 00$ \\
DWI & 3,000 & 89 & 230 & $1.2 \times 1.2 \times 5$ & no & $01: 35$ \\
Sequence $@ 3$ & $\mathrm{~T}$ & & & & & \\
SWI & 27 & 20 & 230 & $0.9 \times 0.9 \times 2$ & 15 & $02: 59$ \\
DSCE & 1,400 & 29 & 230 & $1.8 \times 1.8 \times 5$ & 90 & $01: 59$ \\
DWI & 3,500 & 89 & 230 & $1.8 \times 1.8 \times 4$ & no & $01: 15$ \\
\hline
\end{tabular}

$100 \%$, voxel size $1.8 \times 1.8 \times 5.0 \mathrm{~mm}$, flip angle $90^{\circ}$, acquisition time 1:59 min. DWI parameters were TR 3,500 ms, TE $89 \mathrm{~ms}$, number of averages 4 , FoV read $230 \mathrm{~ms}$, FoV phase $100 \%$, voxel size $1.8 \times 1.8 \times 4.0 \mathrm{~mm}$, acquisition time 1:15 min (Table 1). For both scanners tracer concentrationtime curves of the perfusion sequence were analysed using
Siemens workstations to obtain parametric colour-coded maps of MTT, time-to-peak (TTP), relative cerebral blood flow (rCBF) and relative cerebral blood volume (rCBV). Further, the SWI and minimum intensity projections (mIP) images were generated automatically by the scanner software.
Fig. 1 22-year-old woman with MELAS syndrome and acute global aphasia. a In SWI pseudodiminished cortical veins are seen temporal left. b In association subtle left cortical diffusion restriction and a focal hyperperfusion with $\mathbf{c}$ MTT shortening and $\mathbf{d}$ increased $\mathrm{rCBF}$ (see Table 2, patient 3)

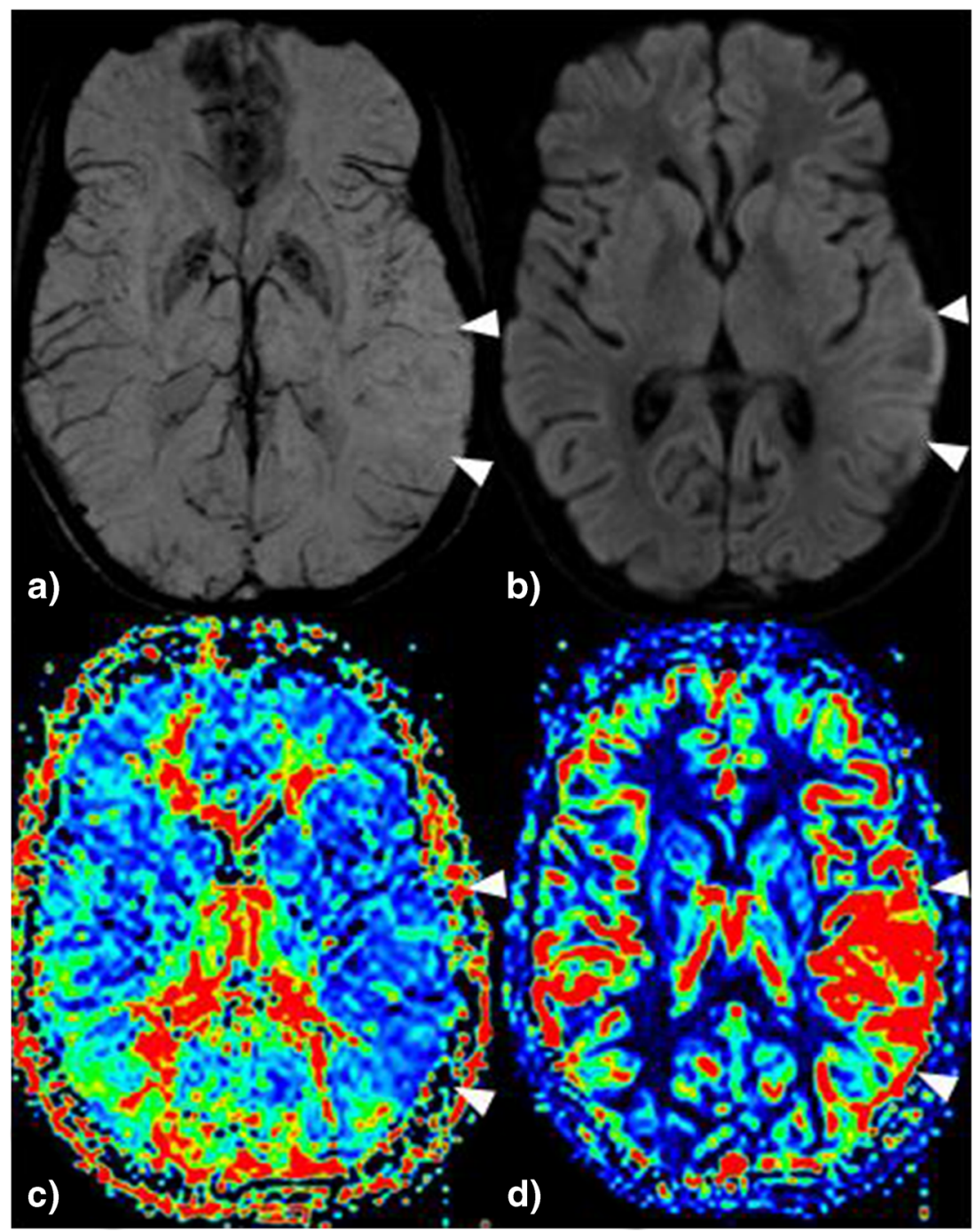


Fig. 2 4-year-old girl with fever seizure and clinical status epilepticus. a Pseudo-narrowed or pseudo-diminished cortical veins are found temporal-parietal and occipital left without diffusion restriction (b, but hyperperfusion in MTT (shortened) and rCBF (increased), see $\mathbf{c}$ and $\mathbf{d}$; (see Table 2, patient 5)

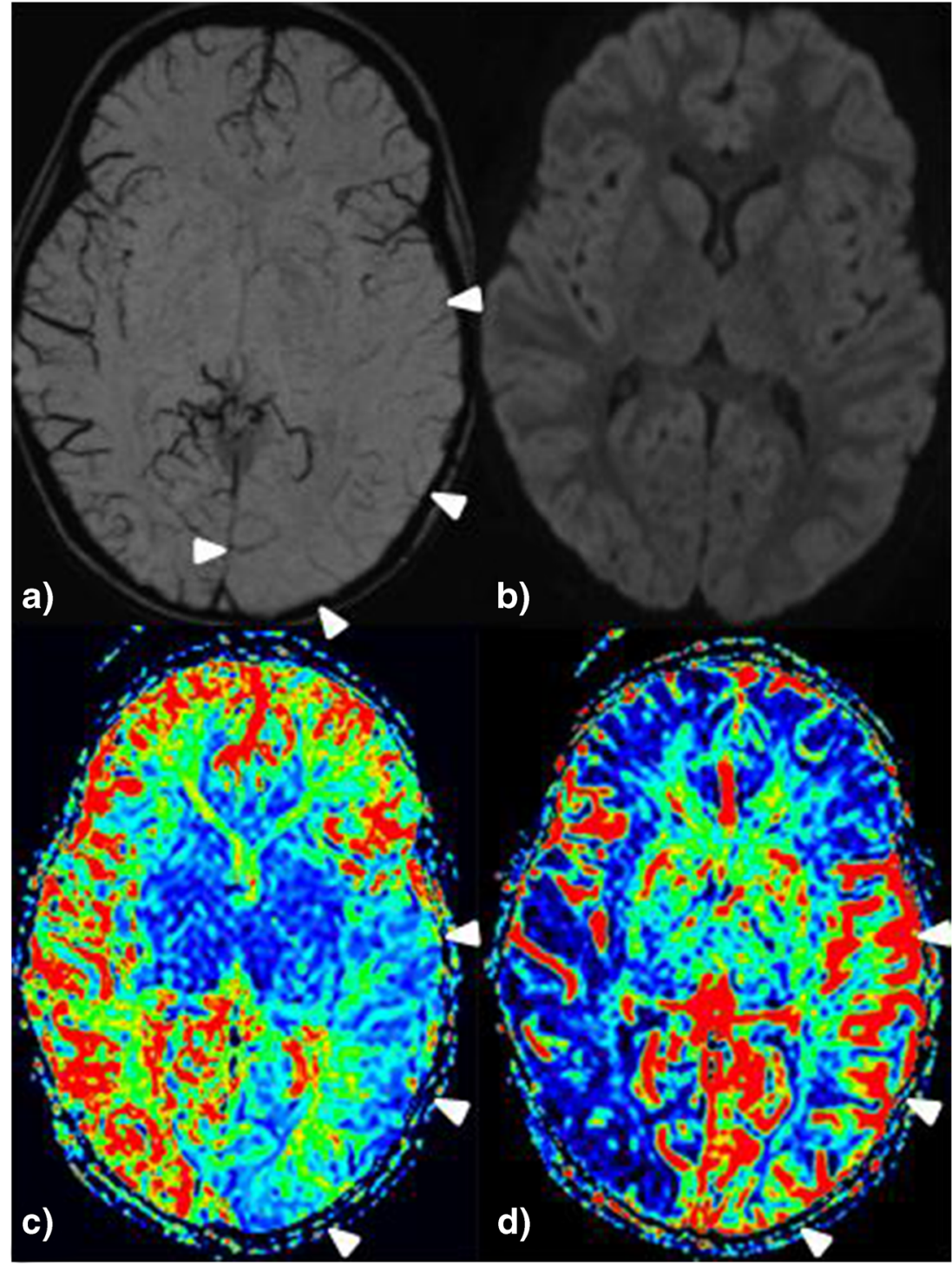

Data analysis

To evaluate MRI sequences, two neuroradiologists were blinded to patient history, except for the information of a current clinically and/or electroclinically confirmed SE and a focal parenchymal area with decreased MTT, without knowledge of the exact location or the focal neurologic signs if present. All MRI sequences were evaluated separately by the neuroradiologists on our picture archiving and communication system (PACS) in a standardized order, first SWI, then rCBF and MTT images, and last DWI images. If cortical veins in SWI were undetectable or less visible in a parenchymal area compared to the cortical veins of the opposite hemisphere the area was noted as positive.

After visual evaluation of DSC data (colour-coded perfusion maps), areas of shortened MTT, increased rCBF and finally areas with diffusion restriction in DWI images were noted.
EEG results of the ten patients with EEG were analysed, and if epileptiform signals (epileptiform discharges (ED), periodic lateralized epileptiform discharges (PLED) or signs compatible with postictal changes (focal or general slowing)) occurred, the region with these alterations was noted in the same manner. Since all findings were positive (pathological) or negative (normal) we used percentage values for description in this small sample without statistical analysis.

Finally all the observed pathological SWI, DWI and DSC findings and the EEG findings were compared to each other and the clinical findings.

\section{Results}

Twelve patients fulfilled the inclusion criteria. In six patients NCSE was confirmed with EEG, all with focal ictal findings. 
Among the remaining six patients with CSE, four underwent EEG within a mean interval time of $21 \mathrm{~h} 8 \mathrm{~min}$, one showed ictal and three postictal EEG changes. In all 12 patients, a focal area with side asymmetrical calibre of cortical veins in the SWI sequence was found, indicating pseudo-narrowing. These areas were associated in all 12 patients with focal hyperperfusion (shortened MTT and increased rCBF, see Fig. 1). Six patients out of $12(50 \%)$ showed a cortical diffusion restriction within the same anatomical territory (see Figs. 2 and 3 as example), five of them with corresponding clinical symptoms (hemiparesis or aphasia). The other six patients had no cortical diffusion restriction. Two patients showed an additional diffusion restriction in the pulvinar of the thalamus, with no correlation in the SWI or perfusion sequences.

Six patients had EEG confirmed NCSE (four with epileptiform discharge, ED; and two with periodic lateralized epileptiform discharges, PLED). The localization of all areas with epileptiform signals correlated with the focal disturbed areas in SWI and DSC.

Two of the six patients with clinically confirmed CSE did not undergo an EEG examination. The other four underwent
EEG. Epileptiform discharges were only detected in one patient (PLED), which was regionally associated with pseudonarrowed veins in SWI. The remaining three showed postictal findings in EEG with generalized slowing in one patient and focal slowing in two patients. In one of the two patients with focal wave slowing, postictal changes were again in the same localization of pseudo-narrowed cortical veins and hyperperfusion in SWI and DSC images, respectively. Besides seizure in CSE, the most frequent neurological symptoms were hemiparesis found in five patients and/or aphasia found in three patients. Again, areas of pathological SWI and DSC findings were associated with the parenchymal areas responsible for these two symptoms in all eight patients (Table 2).

\section{Discussion}

Pathophysiological changes in seizures are well described. SE causes excessive neuronal activity in the early stage and concomitant perfusion increases as a compensatory
Fig. 3 4-year-old girl with fever seizure, Todd paresis left hemisyndrome. a Pseudonarrowed or pseudo-diminished cortical veins are found frontal right with $\mathbf{b}$ correlating diffusion restriction and $\mathbf{c}$ hyperperfusion in the MTT (shortened) and $\mathbf{d}$ CBF maps (increased) (see Table 2, patient 4)

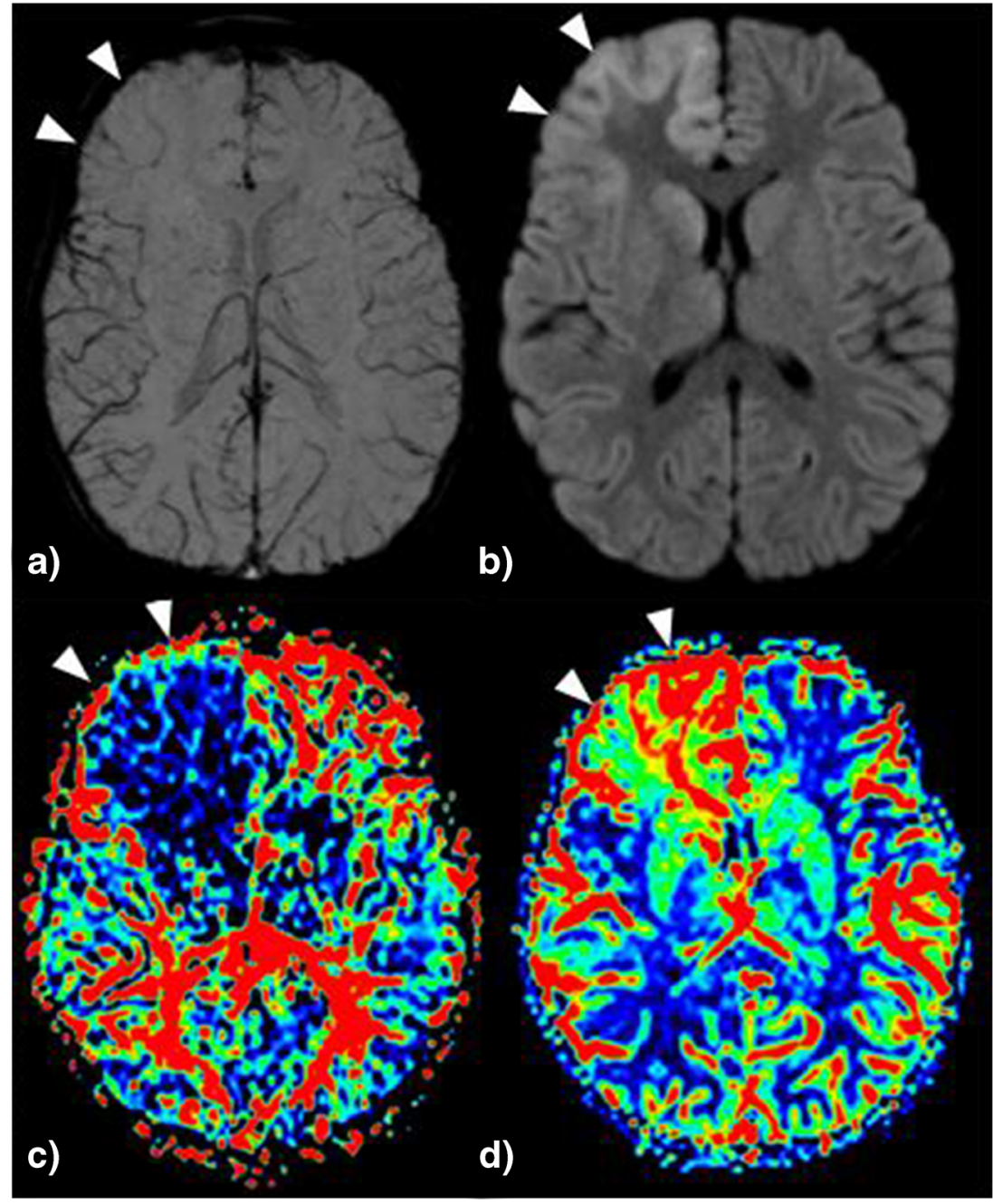




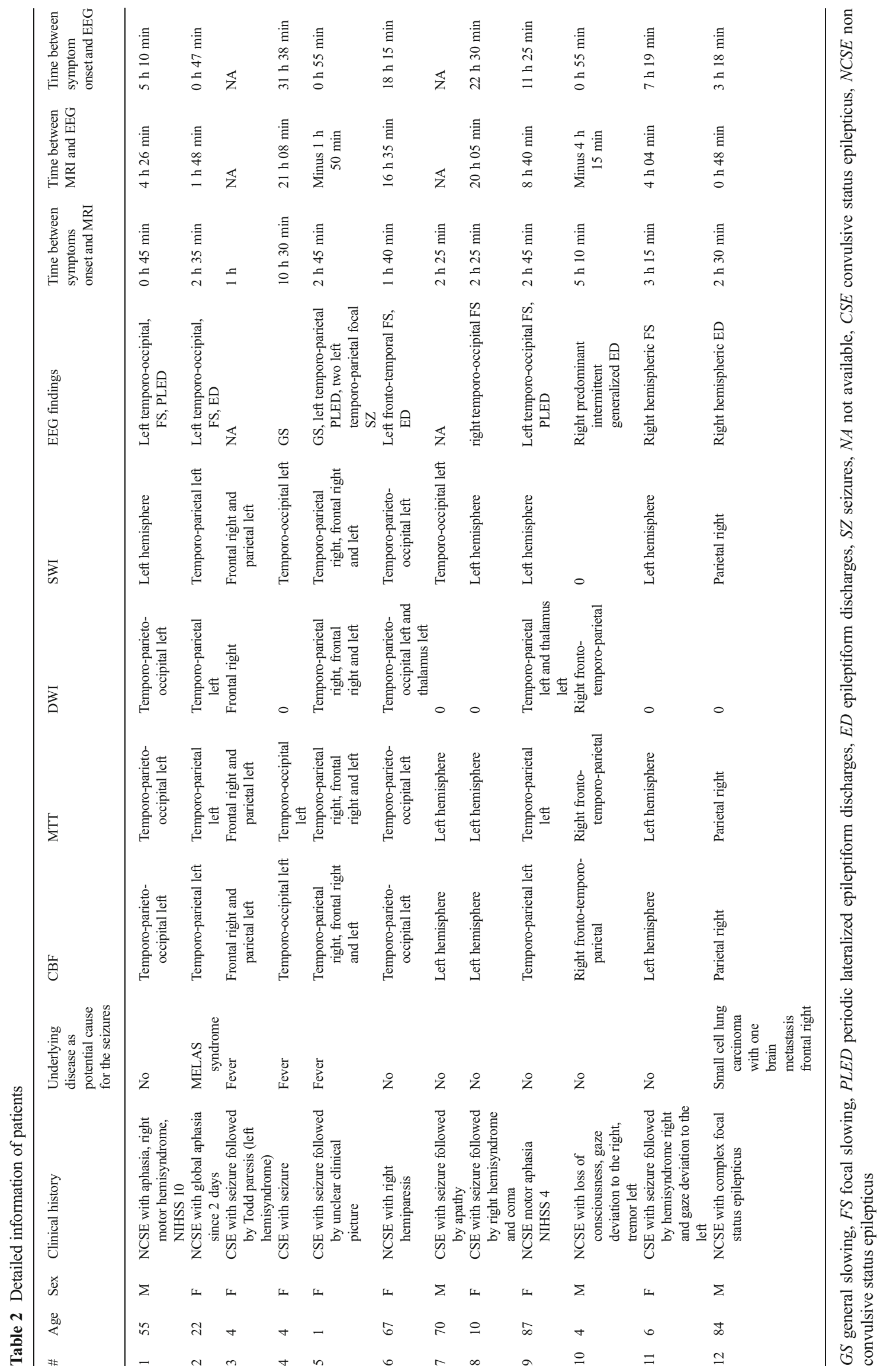


mechanism to the increased metabolic demand, which results in higher levels of oxygenated haemoglobin [25-28].

Consequently this underlying pathophysiological mechanism leads to a net increase of oxyhaemoglobin supply, exceeding the demand, resulting in a net decrease in deoxyhaemoglobin content in the cortical veins. In studies investigating SWI, it is assumed that changes in venous deoxyhaemoglobin levels are proportional to changes of paramagnetic venous properties, resulting in pseudo-calibre changes of the affected cortical veins [17, 20,21].

The observations of this study suggest an association between lower deoxyhaemoglobin levels in cortical veins and increased focal cerebral perfusion. In our retrospective analysis, pseudo-narrowing of cortical veins and ictal or postictal findings in EEG were in the same parenchymal area in eight out of ten patients (six patients with focal epileptogenic discharges and two with postictal focal wave slowing). The remaining two showed a generalized EEG slowing and a focal EEG slowing in the opposite hemisphere, respectively. Since EEGs were performed more than $4 \mathrm{~h}$ before and $20 \mathrm{~h}$ after MRI examination, and SE and NCSE are evolving disorders with highly dynamic changes affecting both clinical and electroencephalographic features [1], we assume that the epileptiform discharges had been widespread or multifocal in these subjects (see Table 2). In eight of eight patients with neurological symptoms of hemiparesis or aphasia, the responsible parenchymal areas correlated with the areas showing focal pseudo-narrowed cortical veins in SWI and focal hyperperfusion in DSC.

Overall, these results suggest that SWI has the potential for detecting ictal areas in early SE by the appearance of pseudonarrowed or pseudo-diminished cortical veins in the affected areas, since pseudo-narrowed cortical veins in SWI were associated in all patients with parenchymal hyperperfusion by location in terms of focal decreased MTT and increased rCBF.

Diffusion restriction was present in only six of 12 patients, so that in our small sample, SWI had a higher detection rate than DWI for a supposed ictal region (for summary see Table 3).

Table 3 Summarized data of patients

\begin{tabular}{lc}
\hline Number of patients & 12 \\
Mean age (years) & 34.5 \\
Sex (female/male) & $8 / 4$ \\
SWI (\%) & 100 \\
MTT (\%) & 100 \\
CBF (\%) & 100 \\
DWI (\%) & 50 \\
EEG findings (10 patients) (\%) & 80 \\
\hline
\end{tabular}

SWI focal pseudo-narrowed cortical veins due to hyperperfusion, MTT associated focal hyperperfusion (MTT decrease) in colour-coded perfusion-maps, $C B F$ associated focal hyperperfusion (CBF increase) in colourcoded perfusion maps, $E E G$ associated focal ictal or postictal findings
Both diffusion restriction and the neurological symptoms of hemiparesis and aphasia are typical findings in acute stroke, whereas a prominent cortical vein appearance in one vascular territory can be found in SWI [18, 21]. NCSE is often underdiagnosed [5] and can clinically mimic an acute stroke or hemiplegic migraine and vice versa. Therefore, SWI may serve as a practical tool to further differentiate between these two pathologies, since the appearance of cortical veins may be the opposite: pseudo-diminished in case of hyperperfusion versus pseudo-prominent cortical veins in case of hypoperfusion. Additionally, in SE the pathological appearance of cortical veins may be in more than one vascular territory.

Our findings corroborate with findings in a patient recently described in a case report [24], where the whole right hemisphere was hyperperfused and cortical veins in SWI were pseudo-diminished during assumed NCSE.

Apart from this case report of Lee et al., very few data have been published about the phenomenon of pseudo-narrowed or pseudo-diminished cortical veins in SWI $[29,30]$. SWI is a very sensitive technique for the detection of intravascular venous deoxygenated blood due to ischemia [31, 32]. Especially in stroke patients, pseudo-prominent cortical veins can be frequently seen in hypoperfused areas [17, 18, 20], caused by a disproportion between oxygen supply and demand resulting in a higher intravenous deoxyhaemoglobin content. Since in our study mainly small calibred or diminished cortical veins were detected in hyperperfused areas, this corroborates the thesis of an association between the extent of cortical vein appearance and deoxyhaemoglobin levels, inversely proportional to cerebral blood flow. This study has some limitations. First, the number of subjects, 12, is small, making further studies necessary with a larger number of patients to confirm our results. A prospective study design, where EEG is performed temporally close to MRI examination would additionally help in validating our results. Furthermore, we selected patients with SE or NCSE having a focally decreased MTT in perfusion imaging. This might lead to selection bias, since potential patients with postictal focal changes like hypoperfusion and increased MTT were eliminated. Gelfand et al. reported that MTT can be prolonged focally after seizure indicating a postictal condition with hypoperfusion [33]. As a consequence deoxyhaemoglobin levels are higher with prominent cortical vein appearance. A recently published case report [34] described three children in whom this phenomenon of focally prominent cortical veins in SWI was associated with epileptic focus localization in acute stage of epileptic encephalopathy. The authors suspected a higher deoxyhaemoglobin concentration. Therefore, caution is advised with the differential diagnosis of ischemic stroke, where a similar perfusion pattern can occur. Future studies should enrol patients with electroclinically confirmed SE/NCSE without regard to focal hyperperfusion. Another bias might be that five of 12 patients were intubated, since oxygen supply can generally reduce 
appearance of cortical veins [35]. However, since in both intubated and non-intubated patients the area with focally reduced cortical vein appearance was surrounded by normal cortical vein appearance, this effect should be negligible.

In summary this study demonstrates an association of focal pseudo-narrowed or pseudo-diminished cortical veins in SWI and focal hyperperfusion in DSC with ictal and postictal signals in EEG, clinical findings and focal diffusion restriction in DWI in this small sample of patients presenting with SE or NCSE. The SWI findings can be explained by a decreased amount of deoxygenated blood and therefore lowered paramagnetic properties due to hyperperfusion of the ictal region. Therefore as a non-contrast sequence, SWI might be useful for detection of a focal ictal area in SE/NCSE.

Acknowledgements The scientific guarantor of this publication is Dr. Rajeev Kumar Verma. The authors of this manuscript declare no relationships with any companies whose products or services may be related to the subject matter of the article. The authors state that this work has not received any funding. No complex statistical methods were necessary for this paper. Institutional review board approval was obtained. Written informed consent was not required for this study because of the retrospective design of the study. Methodology: retrospective, cross-sectional study, performed at one institution.

\section{References}

1. Meierkord H, Holtkamp M (2007) Non-convulsive status epilepticus in adults: clinical forms and treatment. Lancet Neurol 6:329-339

2. Leppik IE (1993) Status epilepticus in the treatment of epilepsy: principles and practice. Lee and Febiger, Philadelphia

3. Shneker BF, Fountain NB (2003) Assessment of acute morbidity and mortality in nonconvulsive status epilepticus. Neurology 61:10661073

4. Maganti R, Gerber P, Drees C, Chung S (2008) Nonconvulsive status epilepticus. Epilepsy Behav 12:572-586

5. Foreman B, Hirsch LJ (2012) Epilepsy emergencies: diagnosis and management. Neurol Clin 30:11-41, vii

6. El-Koussy M, Mathis J, Lovblad KO, Stepper F, Kiefer C, Schroth G (2002) Focal status epilepticus: follow-up by perfusion- and diffusion MRI. Eur Radiol 12:568-574

7. Toledo M, Munuera J, Sueiras M, Rovira R, Alvarez-Sabin J, Rovira A (2008) MRI findings in aphasic status epilepticus. Epilepsia 49: $1465-1469$

8. Hauf M, Slotboom J, Nirkko A, von Bredow F, Ozdoba C, Wiest R (2009) Cortical regional hyperperfusion in nonconvulsive status epilepticus measured by dynamic brain perfusion CT. AJNR Am J Neuroradiol 30:693-698

9. Masterson K, Vargas MI, Delavelle J (2009) Postictal deficit mimicking stroke: role of perfusion CT. J Neuroradiol 36:48-51

10. Szabo K, Poepel A, Pohlmann-Eden B et al (2005) Diffusionweighted and perfusion MRI demonstrates parenchymal changes in complex partial status epilepticus. Brain 128: 1369-1376

11. Hauf M, Wiest R, Schindler K et al (2013) Common mechanisms of auditory hallucinations-perfusion studies in epilepsy. Psychiatry Res 211:268-270
12. Canas N, Breia P, Soares P et al (2010) The electroclinicalimagiological spectrum and long-term outcome of transient periictal MRI abnormalities. Epilepsy Res 91:240-252

13. Hong KS, Cho YJ, Lee SK, Jeong SW, Kim WK, Oh EJ (2004) Diffusion changes suggesting predominant vasogenic oedema during partial status epilepticus. Seizure 13:317-321

14. Fahrendorf D, Schwindt W, Wolfer J et al (2013) Benefits of contrastenhanced SWI in patients with glioblastoma multiforme. Eur Radiol 23:2868-2879

15. Gramsch C, Goricke SL, Behrens F et al (2013) Isolated cerebral susceptibility artefacts in patients with malignant melanoma: metastasis or not? Eur Radiol 23:2622-2627

16. Mittal S, Wu Z, Neelavalli J, Haacke EM (2009) Susceptibilityweighted imaging: technical aspects and clinical applications, part 2. AJNR Am J Neuroradiol 30:232-252

17. Santhosh K, Kesavadas C, Thomas B, Gupta AK, Thamburaj K, Kapilamoorthy TR (2009) Susceptibility weighted imaging: a new tool in magnetic resonance imaging of stroke. Clin Radiol 64:74-83

18. Hermier M, Nighoghossian N (2004) Contribution of susceptibilityweighted imaging to acute stroke assessment. Stroke 35:1989-1994

19. Saini J, Kesavadas C, Thomas B et al (2009) Susceptibility weighted imaging in the diagnostic evaluation of patients with intractable epilepsy. Epilepsia 50:1462-1473

20. Kao HW, Tsai FY, Hasso AN (2012) Predicting stroke evolution: comparison of susceptibility-weighted MR imaging with MR perfusion. Eur Radiol 22:1397-1403

21. Huang P, Chen CH, Lin WC, Lin RT, Khor GT, Liu CK (2012) Clinical applications of susceptibility weighted imaging in patients with major stroke. J Neurol 259:1426-1432

22. Verschuuren S, Poretti A, Buerki S, Lequin MH, Huisman TA (2012) Susceptibility-weighted imaging of the pediatric brain. AJR Am J Roentgenol 198:W440-W449

23. Bosemani T, Burton VJ, Felling RJ et al (2013) Pediatric hemiplegic migraine: role of multiple MRI techniques in evaluation of reversible hypoperfusion. Cephalalgia. doi:10.1177/0333102413509432

24. Lee YJ, Shon YM, Yoo WJ, Jung SL, Kim BS, Ahn KJ (2013) Diminished visibility of cerebral venous vasculature in subclinical status epilepticus by susceptibility-weighted imaging: a case report. Clin Neuroradiol. doi:10.1007/s00062-013-0204-3

25. Zhao M, Suh M, Ma H, Perry C, Geneslaw A, Schwartz TH (2007) Focal increases in perfusion and decreases in hemoglobin oxygenation precede seizure onset in spontaneous human epilepsy. Epilepsia 48:2059-2067

26. Wasterlain CG, Fujikawa DG, Penix L, Sankar R (1993) Pathophysiological mechanisms of brain damage from status epilepticus. Epilepsia 34:S37-S53

27. Unrath A, Muller HP, Ludolph AC, Kassubek J (2012) Reversible cortical diffusion restriction, hyperperfusion and T2-hyperintensity caused by two different types of epileptic seizure. Clin Neuroradiol 22:239-243

28. Xing W, Wang X, Xie F, Liao W (2013) Application of dynamic susceptibility contrast-enhanced perfusion in temporal lobe epilepsy. Acta Radiol 54:107-112

29. Ge Y, Zohrabian VM, Osa EO et al (2009) Diminished visibility of cerebral venous vasculature in multiple sclerosis by susceptibility-weighted imaging at 3.0 Tesla. J Magn Reson Imaging 29:1190-1194

30. Tsui YK, Tsai FY, Hasso AN, Greensite F, Nguyen BV (2009) Susceptibility-weighted imaging for differential diagnosis of cerebral vascular pathology: a pictorial review. J Neurol Sci 287:7-16

31. Thomas B, Somasundaram S, Thamburaj K et al (2008) Clinical applications of susceptibility weighted MR imaging of the brain - a pictorial review. Neuroradiology 50:105-116 
32. Tong KA, Ashwal S, Obenaus A, Nickerson JP, Kido D, Haacke EM (2008) Susceptibility-weighted MR imaging: a review of clinical applications in children. AJNR Am J Neuroradiol 29:9-17

33. Gelfand JM, Wintermark M, Josephson SA (2010) Cerebral perfusion-CT patterns following seizure. Eur J Neurol 17:594-601
34. Iwasaki H, Takeda T, Ito T et al (2014) The use of susceptibilityweighted imaging for epileptic focus localization in acute-stage pediatric encephalopathy: a case report. Pediatr Neurol 50:171-176

35. Bosemani T, Verschuuren SI, Poretti A, Huisman TA (2013) Pitfalls in susceptibility-weighted imaging of the pediatric brain. $\mathrm{J}$ Neuroimaging. doi:10.1111/jon.12051 